\section{Emergency department point-of-care ultrasonography improves time to pericardiocentesis for clinically significant effusions}

\author{
Evan Avraham Alpert ${ }^{1 *}$, Uri Amit ${ }^{2}$, Larisa Guranda ${ }^{3}$, Rafea Mahagna ${ }^{1}$, \\ Shamai A. Grossman ${ }^{4}$, Ariel Bentancur ${ }^{1}$ \\ 'Department of Emergency Medicine, Sheba Medical Center, Tel Hashomer, Israel \\ ${ }^{2}$ Neufeld Cardiac Research Institute, Sheba Medical Center, Tel-Aviv University, Tel Hashomer, Israel \\ ${ }^{3}$ Department of Radiology, Sheba Medical Center, Tel Hashomer, Israel \\ ${ }^{4}$ Department of Emergency Medicine, Beth Israel Deaconess Medical Center, Harvard Medical School, \\ Boston, MA, USA
}

Objective Our objective was to determine the utility of point-of-care ultrasound (POCUS) to identify and guide treatment of tamponade or clinically significant pericardial effusions in the emergency department (ED).

Methods This was a retrospective cohort study of non-trauma patients who were diagnosed with large pericardial effusions or tamponade by the ED physician using POCUS. The control group was composed of those patients later diagnosed on the medical wards or incidentally in the ED by other means such as a computed tomography. The following data were abstracted from the patient's file: demographics, medical background, electrocardiogram results, chest radiograph readings, echocardiogram results, and patient outcomes.

Results There were 18 patients in the POCUS arm and 55 in the control group. The POCUS arm had a decreased time to pericardiocentesis ( 11.3 vs. 70.2 hours, $P=0.055$ ) as well as a shorter length of stay ( 5.1 vs. 7.0 days, $P=0.222$ ). A decreased volume of pericardial fluid was drained (661 vs. $826 \mathrm{~mL}, \mathrm{P}=0.139$ ) in the group diagnosed by POCUS.

Conclusion This study suggests that POCUS may effectively identify pericardial effusions and guide appropriate treatment, leading to a decreased time to pericardiocentesis and decreased length of hospital stay. Pericardial tamponade or a large pericardial effusion should be considered in all patients presenting to the ED with clinical, radiographic, or electrocardiographic signs of cardiovascular compromise.

Keywords Cardiac tamponade; Ultrasonography; Emergencies
elSSN: 2383-4625

Received: 29 July 2016

Revised: 8 March 2017

Accepted: 15 March 2017

Correspondence to:

Evan Avraham Alpert

Department of Emergency Medicine, Shaare Zedek Medical Center, Shmu'el Bait St 12, Jerusalem, Israel, 9103102 , Israel

E-mail: evanavrahamalpert@gmail. com

*Current affiliation: Department of Emergency Medicine, Shaare Zedek Medical Center, Jerusalem, Israel

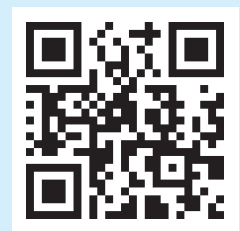

How to cite this article:

Alpert EA, Amit U, Guranda L, Mahagna R, Grossman SA, Bentancur A. Emergency department point-of-care ultrasonography improves time to pericardiocentesis for clinically significant effusions. Clin Exp Emerg Med 2017;4(3):128-132.

This is an Open Access article distributed under the terms of the Creative Commons Attribution Non-Commercial License (http:// creativecommons.org/licenses/by-nc/4.0/). 


Wapsule
Wummary is already known
Point-of-care ultrasound can be used by the emergency physician to identify large pericardial effusions and tampon-
ade.
$\begin{aligned} & \text { What is new in the current study } \\ & \text { This study quantifies the effect of early diagnosis by the emergency physician of large pericardial effusions or tampon- } \\ & \text { ade in terms of decreased time to pericardiocentesis and decreased length of stay. }\end{aligned}$

\section{INTRODUCTION}

During the resuscitation of a patient in shock or cardiac arrest, rapid diagnosis and treatment of reversible causes such as cardiac tamponade is critical, as delays may lead to increased morbidity and mortality. ${ }^{1}$ Point-of-care ultrasound (POCUS) may play a primary role in the care of such patients. ${ }^{2-5}$ The first model curriculum for POCUS was published by Mateer in 1994. ${ }^{6}$ The basic focus of the cardiac component was to evaluate the presence of cardiac motion and pericardial fluid. Mandavia et al. ${ }^{7}$ demonstrated that through this approach, emergency physicians (EPs) were able to identify a pericardial effusion with a sensitivity of $96 \%$ and specificity of $98 \%$. However, they did not determine their ability to diagnose tamponade or discuss the outcomes of their findings.

The objective of this study was to evaluate the time to diagnosis and outcomes of patients with tamponade or large pericardial effusions that were identified by EPs using POCUS in comparison with patients in whom emergency department (ED) ultrasound was not performed.

\section{METHODS}

\section{Study design and setting}

This retrospective cohort study was conducted in a large urban academic referral center with an annual ED census of 120,000. From October 2007 to February 2012, we studied consecutive patients presenting to the ED with a diagnosis-related group at discharge of tamponade or pericardial effusion. Institutional review board approval (9311-12), waiving informed consent, was obtained before initiation of the study.

\section{Selection of participants}

Inclusion criteria for the POCUS group were non-trauma patients identified in the ED using POCUS to determine the presence of clinically significant pericardial effusion (defined as an effusion that would lead to a decision to refer the patient to a cardiologist or cardiothoracic surgeon for pericardiocentesis or operative repair). Although there were no strict criteria, such a decision could include size of effusion or right ventricular collapse during diastole. These exams were performed based on the clinical discretion of the individual physician.

All patients with a definitive diagnosis of a large pericardial effusion or tamponade were identified using the hospital database. The non-POCUS group was composed of those patients who did not have POCUS in the ED but later were diagnosed on the medical wards or incidentally in the ED by other means such as a computed tomography. Trauma patients were excluded.

\section{POCUS protocol}

Six physicians recorded the ultrasounds and provided coverage from 08:00 to 24:00. Five were board certified in emergency medicine, and one was a fellow in emergency medicine. All trained in emergency ultrasound according to the curriculum of the Israeli Association of Emergency Medicine. The views obtained included the subxyphoid view, apical four-chamber view, and parasternal long-axis view. Ultrasound machines were the SonoSite Micromaxx (SonoSite, Bothell, WA, USA), the LOGICe (GE Healthcare, Waukesha, WI, USA), and the Philips CX50 (Philips Healthcare, Amsterdam, The Netherlands).

\section{Data collection and processing}

Data over the course of the 4.5-year study period was entered into a Microsoft Excel (Redmond, WA, USA) template. Demographics, medical background information, and electrocardiogram (ECG), chest radiography (CXR), ultrasound, and official echocardiogram results were recorded along with patient outcomes. CXR results were recorded based on the official radiologist reading in the picture archiving and communication system. ECG readings were based on results recorded in the MUSE Cardiology Informa- 
tion System (GE Healthcare). The collected data included treatment methods (pericardiocentesis, operative repair, or medical treatment) and results (probable etiology of pericardial fluid, length of hospital stay, and status at hospital discharge). The door-to-pericardiocentesis time was recorded from the Horizon Cardiology Web Cardiovascular Information System database (McKesson, San Francisco, CA, USA) and from the database in the cardiology department in our institution.

\section{Outcome measures}

The primary outcomes were door-to-pericardiocentesis time, amount of pericardiocentesis fluid, and length of hospital stay. Mortality served as a secondary outcome.

\section{Statistical analysis}

Statistical analysis was performed using IBM SPSS Statistics ver. 19 (IBM Corp., Armonk, NY, USA). Categorical variables are expressed as percentages and continuous variables as mean \pm standard deviation. Comparisons of variables were performed using the chi-square test for categorical variables and Student's t-test for continuous variables. A P-value of $<0.05$ was considered statistically significant for all calculations.

\section{RESULTS}

Out of an estimated 500,000 ED visits during this study period and with an estimated 1,000 POCUS studies performed, 18 patients were diagnosed in the ED by EPs as having significant pericardial effusions by POCUS while 55 were diagnosed by other means (Fig. 1). Demographic information, triage complaints, and vital signs are depicted in Table 1. Although the demographics

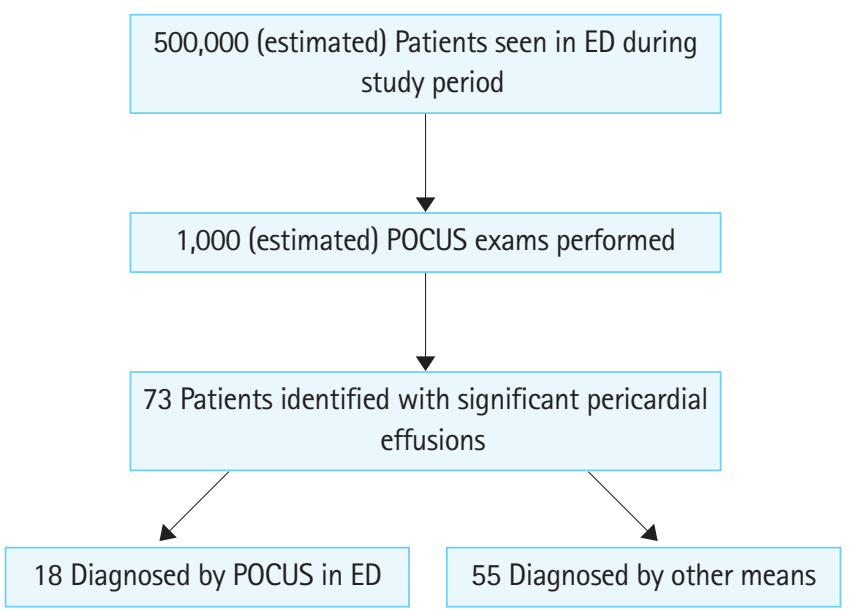

Fig. 1. Study participants. ED, emergency department; POCUS, pointof-care ultrasound. between the two groups were similar, the POCUS group had a significantly lower systolic blood pressure (117.6 \pm 28.9 vs. $134.8 \pm$ $21.9 \mathrm{mmHg}, \mathrm{P}=0.013$ ) and more patients presenting with syncope (3 vs. $0, P=0.006$ ).

Table 2 depicts ECG and CXR findings. More patients in the study group had low voltage on their ECG (41.2\% vs. $20.7 \%$, $P=0.136$ ). Both groups of patients had an enlarged cardiac silhouette, but more patients in the control group had findings of infiltrate or effusion (71.7\% vs. $17.6 \%, \mathrm{P}<0.001)$ on CXR.

The characteristics and etiologies of the effusions as well as the study outcomes are shown in Table 3. Malignancy was the most frequent cause of pericardial effusion in both groups. Other causes were much less common. The door-to-pericardiocentesis time was decreased (11.3 vs. 70.2 hours, $P=0.055$ ) in the intervention group. Although not statistically significant, the length of

Table 1. Demographic and triage data

\begin{tabular}{lccc}
\hline Characteristics & $\begin{array}{c}\text { POCUS group } \\
(\mathrm{n}=18)\end{array}$ & $\begin{array}{c}\text { Non-POCUS } \\
\text { group }(\mathrm{n}=55)\end{array}$ & P-value \\
\hline Demographic information & & & \\
$\quad$ Age (yr) & $65.6 \pm 17.7$ & $61.5 \pm 18.5$ & 0.402 \\
Sex (female) & $11(61.1)$ & $26(47.3)$ & 0.308 \\
Triage vital signs & & & \\
Systolic blood pressure $(\mathrm{mmHg})$ & $117.6 \pm 28.9$ & $134.8 \pm 21.9$ & 0.013 \\
Pulse rate & $99.1 \pm 21.4$ & $98.0 \pm 19.8$ & 0.840 \\
Temperature ( $\left.{ }^{\circ} \mathrm{C}\right)$ & $36.6 \pm 0.6$ & $36.8 \pm 0.7$ & 0.458 \\
Oxygen saturation $(\%)$ & $93.5 \pm 3.1$ & $93.5 \pm 5.4$ & 0.962 \\
Triage complaints & & & \\
Chest pain & $6(33.3)$ & $10(23.3)$ & 0.414 \\
Dyspnea & $8(44.4)$ & $22(51.2)$ & 0.632 \\
Weakness or dizziness & $6(33.3)$ & $13(30.2)$ & 0.811 \\
Abdominal or back pain & $1(5.6)$ & $6(14.0)$ & 0.348 \\
Fever & $1(5.6)$ & $6(14.0)$ & 0.348 \\
Cough & $1(5.6)$ & $4(9.3)$ & 0.627 \\
Syncope & $3(16.7)$ & $0(0)$ & 0.006 \\
\hline
\end{tabular}

Values are presented as mean \pm standard deviation or number $(\%)$. POCUS, point-of-care ultrasound.

Table 2. Electrocardiographic and chest radiograph findings

\begin{tabular}{lccr}
\hline Characteristics & $\begin{array}{c}\text { POCUS } \\
\text { group }\end{array}$ & $\begin{array}{c}\text { Non-POCUS } \\
\text { group }\end{array}$ & P-value \\
\hline $\begin{array}{l}\text { Electrocardiographic findings } \\
\text { Sinus tachycardia }\end{array}$ & $7(41.2)$ & $11(37.9)$ & 0.828 \\
Low voltage & $7(41.2)$ & $6(20.7)$ & 0.136 \\
ST abnormalities & $5(29.4)$ & $11(37.9)$ & 0.558 \\
Atrial fibrillation & $2(11.8)$ & $3(10.3)$ & 0.881 \\
Myocardial infarction & $3(17.6)$ & $5(17.2)$ & 0.972 \\
Pacemaker & $1(5.9)$ & $2(6.9)$ & 0.893 \\
Chest radiograph findings & & & \\
$\quad$ Enlarged cardiac silhouette & $12(70.6)$ & $38(86.4)$ & 0.151 \\
Infiltrate or effusion & $3(17.6)$ & $33(71.7)$ & $<0.001$ \\
\hline
\end{tabular}

Values are presented as number (\%). POCUS, point-of-care ultrasound. 
Table 3. Outcome data

\begin{tabular}{lccc}
\hline Characteristics & $\begin{array}{c}\text { POCUS } \\
\text { group }\end{array}$ & $\begin{array}{c}\text { Non-POCUS } \\
\text { group }\end{array}$ & P-value \\
\hline Suspected etiology & & & \\
$\quad$ Malignancy & $7(46.7)$ & $21(72.4)$ & 0.092 \\
Viral & $2(13.3)$ & $1(3.4)$ & 0.218 \\
Procedure related & $2(13.3)$ & $1(3.4)$ & 0.218 \\
Coumadin & $1(6.7)$ & $3(10.3)$ & 0.687 \\
Free wall rupture & $1(6.7)$ & $0(0)$ & 0.160 \\
Post-pericardiotomy syndrome & $1(6.7)$ & $1(3.4)$ & 0.627 \\
Renal failure & $1(6.7)$ & $0(0)$ & 0.160 \\
Outcomes & & & \\
$\quad$ Time to pericardiocentesis (hr) & $11.3 \pm 13.8$ & $70.2 \pm 126.2$ & 0.055 \\
Amount of fluid drained (mL) & $660.6 \pm 339.9$ & $825.8 \pm 409.2$ & 0.139 \\
Length of hospital stay (day) & $5.0 \pm 0.6$ & $7.0 \pm 0.9$ & 0.222 \\
Death & $1(5.6)$ & $4(7.3)$ & 0.802 \\
\hline
\end{tabular}

Values are presented as number (\%) or mean \pm standard deviation. POCUS, point-of-care ultrasound.

hospital stay was shorter by 2 days in the intervention group (5.1 vs. 7.0 days, $P=0.22$ ). A decreased volume of pericardial fluid was drained (661 vs. $826 \mathrm{~mL}, P=0.14$ ) in the group diagnosed by ED ultrasound.

\section{DISCUSSION}

Our data show that the EP can effectively identify significant pericardial effusions using POCUS. This leads to shorter times before a procedure is performed and potentially shorter hospital stays. This is especially important when an attending echocardiographer is not in the hospital after hours. To the best of our knowledge, based on a search using International Classification of Diseases codes including "tamponade" and "pericardial effusion," no patients that were later identified during hospitalization (the control group) as having a significant pericardial effusion had a previous falsely negative POCUS examination in the ED.

Tamponade is a common life-threatening culprit of cardiac arrest or hypotension, and POCUS in these settings may be invaluable. However, the majority of patients in both of our cohorts were normotensive, similar to what was seen in a previous case series. $^{8}$ The average lower blood pressure in the POCUS group $(117.6 \pm 28.9$ vs. $134.8 \pm 21.9 \mathrm{mmHg}, \mathrm{P}=0.013)$ probably would not in and of itself alert the clinician to suspect tamponade or significant pericardial effusion unless the systolic pressure was below $100 \mathrm{mmHg}$. In addition, the more common finding of syncope alone in the POCUS group probably would not have alerted the clinician to suspect tamponade. Only a minority had hypoxia or tachycardia. In our population, the most common cause of tamponade was malignancy. This is also similar to previous studies of patients on medical or cardiology services. ${ }^{9-11}$
Although sinus tachycardia and low voltage on the ECG may indicate the presence of significant pericardial fluid, these are non-specific findings. The majority of CXRs in both groups were interpreted by the radiologist as indicative of an enlarged heart size. Previous literature cites an enlarged cardiac silhouette as moderately sensitive (71\%) but not specific (41\%) for pericardial effusion. ${ }^{12}$ The pericardial fat stripe, predominantly left-sided pleural effusion, and an increased transverse cardiac diameter were all noted to be more specific than an enlarged cardiac silhouette $(94 \%, 100 \%$, and $80 \%$ specificity, respectively). Our series included one case with a left-sided pleural effusion, but none were noted to have any of the other signs. Interestingly, a significant number of CXRs in the control group had an infiltrate or effusion $(71.7 \%$ vs. $17.6 \%, P<0.001)$, which may have led the ED physician to bypass the differential of tamponade so that these patients were subsequently diagnosed on the wards.

The earlier diagnosis of significant pericardial effusions in the ED therefore appears to be due to a combination of some or all of the following: vital sign abnormalities, clinical chief complaint, and past medical history as well as ECG and/or CXR findings.

We decided to include all patients with significant pericardial effusions and not only pericardial tamponade because this finding can potentially change the patient's management whether by means of pericardiocentesis, operative repair, or medical treatment. The choice of management was often carried out in consultation with cardiology, as pericardial disease produces a spectrum of hemodynamic changes and often there is no black or white decision as to whether a patient requires conservative or invasive management. ${ }^{13,14}$

In a previous series of non-traumatic tamponade, approximately $75 \%$ of patients underwent pericardiocentesis and $10 \%$ died in the hospital. ${ }^{9}$ In our cohort, a higher percentage underwent pericardiocentesis or surgery (82.6\%) with a lower mortality $(4.3 \%)$, suggesting benefit from a more aggressive diagnostic and therapeutic approach.

An emergency medicine literature review revealed individual case reports of cardiac tamponade. ${ }^{15,16}$ By bringing together our data as a case series, we show the broad range of presentations of large pericardial effusions and tamponade and that the EP can be instrumental in identifying patients who often present subtly with life-threatening disease.

Pericardial tamponade or a large pericardial effusion should be considered in all patients presenting to the ED with respiratory symptoms or signs of cardiovascular compromise, especially with a background of malignancy, or when ECG and CXR suggest so.

The major limitation to this study is the lack of clear criteria for the decision of who needs POCUS to identify tamponade or a 
large pericardial effusion, which could have potentially resulted in selection bias. The utility of POCUS, like any medical procedure, will always be somewhat skill-dependent. However, we believe that with a standardized curriculum, POCUS to identify tamponade can be adopted widely throughout EDs. Lastly, this was a single-center study and therefore may not lend itself to generalizability.

This study suggests that bedside POCUS performed by trained EPs may effectively identify significant pericardial effusions and guide appropriate interventional or medical treatment, potentially leading to a decreased time to pericardiocentesis as well as decreased length of hospital stay.

\section{CONFLICT OF INTEREST}

No potential conflict of interest relevant to this article was reported.

\section{REFERENCES}

1. Sebat $F$, Musthafa $A A$, Johnson $D$, et al. Effect of a rapid response system for patients in shock on time to treatment and mortality during 5 years. Crit Care Med 2007;35:2568-75.

2. Rose JS, Bair AE, Mandavia D, Kinser DJ. The UHP ultrasound protocol: a novel ultrasound approach to the empiric evaluation of the undifferentiated hypotensive patient. Am J Emerg Med 2001;19:299-302.

3. Hernandez C, Shuler $K$, Hannan $H$, Sonyika $C$, Likourezos $A$, Marshall J. CAUSE: cardiac arrest ultra-sound exam. A better approach to managing patients in primary non-arrhythmogenic cardiac arrest. Resuscitation 2008;76:198-206.

4. Atkinson PR, McAuley DJ, Kendall RJ, et al. Abdominal and Cardiac Evaluation with Sonography in Shock (ACES): an approach by emergency physicians for the use of ultrasound in patients with undifferentiated hypotension. Emerg Med J 2009;26:87-91.

5. Seif D, Perera P, Mailhot T, Riley D, Mandavia D. Bedside ultra- sound in resuscitation and the rapid ultrasound in shock protocol. Crit Care Res Pract 2012;2012:503254.

6. Mateer J, Plummer D, Heller M, et al. Model curriculum for physician training in emergency ultrasonography. Ann Emerg Med 1994;23:95-102.

7. Mandavia DP, Hoffner RJ, Mahaney K, Henderson SO. Bedside echocardiography by emergency physicians. Ann Emerg Med 2001;38:377-82.

8. Kapoor T, Locurto M, Farina GA, Silverman R. Hypotension is uncommon in patients presenting to the emergency department with non-traumatic cardiac tamponade. J Emerg Med 2012;42:220-6.

9. Cornily JC, Pennec PY, Castellant $P$, et al. Cardiac tamponade in medical patients: a 10-year follow-up survey. Cardiology 2008;111:197-201.

10. Levine MJ, Lorell BH, Diver DJ, Come PC. Implications of echocardiographically assisted diagnosis of pericardial tamponade in contemporary medical patients: detection before hemodynamic embarrassment. J Am Coll Cardiol 1991;17:59-65.

11. Guberman BA, Fowler NO, Engel PJ, Gueron M, Allen JM. Cardiac tamponade in medical patients. Circulation 1981;64: 633-40.

12. Eisenberg MJ, Dunn MM, Kanth N, Gamsu G, Schiller NB. Diagnostic value of chest radiography for pericardial effusion. $J$ Am Coll Cardiol 1993;22:588-93.

13. Shabetai R. Pericardial effusion: haemodynamic spectrum. Heart 2004;90:255-6.

14. Roy CL, Minor MA, Brookhart MA, Choudhry NK. Does this patient with a pericardial effusion have cardiac tamponade? JAMA 2007;297:1810-8.

15. Liang EC, Rossi J, Gharahbaghian L. Cardiac tamponade after minimally invasive coronary artery bypass graft. West J Emerg Med 2011;12:116-7.

16. Leeies M, Weldon E. STEMI stymie: metastatic cancer and cardiac tamponade presenting as inferior STEMI. Ann Emerg Med 2011;57:221-4. 